\title{
In vivo visualization of the facial nerve in patients with acoustic neuroma using diffusion tensor imaging-based fiber tracking
}

\author{
${ }^{*}$ Fei Song, MM, ${ }^{1}$ Yuanzheng Hou, MD, ${ }^{1}$ Guochen Sun, MD, ${ }^{1}$ Xiaolei Chen, MD, ${ }^{1}$ Bainan Xu, MD, ${ }^{1}$ \\ Jason H. Huang, MD, ${ }^{2}$ and Jun Zhang, MD' \\ 'Department of Neurosurgery, PLA General Hospital, Beijing, China; and 2Department of Neurosurgery, Texas A\&M Health \\ Science Center College of Medicine, Bryan, Texas
}

\begin{abstract}
OBJECTIVE Preoperative determination of the facial nerve (FN) course is essential to preserving its function. Neither regular preoperative imaging examination nor intraoperative electrophysiological monitoring is able to determine the exact position of the FN. The diffusion tensor imaging-based fiber tracking (DTI-FT) technique has been widely used for the preoperative noninvasive visualization of the neural fasciculus in the white matter of brain. However, further studies are required to establish its role in the preoperative visualization of the FN in acoustic neuroma surgery. The object of this study is to evaluate the feasibility of using DTI-FT to visualize the FN.
\end{abstract}

METHODS Data from 15 patients with acoustic neuromas were collected using 3-T MRI. The visualized FN course and its position relative to the tumors were determined using DTI-FT with 3D Slicer software. The preoperative visualization results of FN tracking were verified using microscopic observation and electrophysiological monitoring during microsurgery.

RESULTS Preoperative visualization of the FN using DTI-FT was observed in $93.3 \%$ of the patients. However, in $92.9 \%$ of the patients, the $\mathrm{FN}$ visualization results were consistent with the actual surgery.

CONCLUSIONS DTI-FT, in combination with intraoperative FN electrophysiological monitoring, demonstrated improved FN preservation in patients with acoustic neuroma. FN visualization mainly included the facial-vestibular nerve complex of the $\mathrm{FN}$ and vestibular nerve.

http://thejns.org/doi/abs/10.3171/2015.7.JNS142922

KEY WORDS cerebellopontine angle; neuroma; diffusion tensor imaging; facial nerve; fiber tracking; diagnostic and operative techniques

$\mathrm{F}$ OR most large acoustic neuromas that bulge into the cerebellopontine angle, the focus of surgery has seen a gradual shift from total resection of the tumor to the removal of the tumor and retaining the functions of the facial nerve (FN) and cochlear nerve. According to various studies, the anatomical retention rate of the FN is greater than $90 \%$, whereas the functional retention rate is only $60 \%$ to $90 \% .^{15}$

Estimating FN conditions before acoustic neuroma surgery depends on MRI examinations such as the 3D constructive interference in steady state sequence ${ }^{6}$ and the $3 \mathrm{D}$ fast imaging employing steady state acquisition (FIESTA) sequence. These techniques indicate nerve courses with signal comparisons between the nerve fibers, surrounding tissues, and CSF. However, for the FN, the surrounding CSF, bone substance, and tumor-related compression affect its imaging, thereby making identification difficult. In acoustic neuroma surgery, protecting the $\mathrm{FN}$ depends on the surgeon's familiarity with the local anatomy and presurgical assessment of the FN's location relative to the tumor. Continuous electromyography monitoring can be used to determine the spontaneous and inductive facial muscles and detect the FN's course and thus protect and help avoid irreversible FN injury. However, repeated pull-

ABBREVIATIONS DTI-FT = diffusion tensor imaging-based fiber tracking; FA = fractional anisotropy; FIESTA = fast imaging employing steady-state acquisition; FIESTA-C = FIESTA with cycle phase sequences; FN = facial nerve; $I A C=$ internal auditory canal; REZ = root exit zone; ROI = region of interest. SUBMITTED December 24, 2014. ACCEPTED July 15, 2015.

* Fei Song and Dr. Hou contributed equally to this work. 
ing within the resection region causes some injury to the FN. Additionally, various researchers have opined that electrical stimulation also has a certain degree of influence on $\mathrm{FN}$ function. ${ }^{16}$

Diffusion tensor imaging-based fiber tracking (DTIFT) can be used to accurately determine the FN's spatial position, thereby minimizing injury due to pulling, appropriately probing the $\mathrm{FN}$, and reducing $\mathrm{FN}$ injuries caused by electrical stimulation. Additionally, the surgeon's ability to precisely determine the FN's spatial position and protect it determines the FN's anatomical and functional retention rates. DTI-FT has been widely used in tracking and reconstructing neural fasciculus in the white matter, including the $3 \mathrm{D}$ reconstructed pyramidal tract, arcuate fasciculus, and optic radiation, and is clinically applied for intraoperative navigation due to its accuracy. ${ }^{10}$ However, related applications for tracking and reconstructing FNs before acoustic neuroma surgery are still rare.

In this study, we discuss the repeatability of applying DTI-FT to reconstructing the FN under existing clinical conditions in patients with acoustic neuroma. This study also highlighted the comparison between the visualization results of the preoperative FN and the observed FN conditions in order to validate the accuracy of the technology.

\section{Methods \\ Patients}

Sixteen patients (1 patient was excluded due to the lack of intraoperative microscopic video data) from the General Hospital of People's Liberation Army (November 2012 to July 2013) were included in the study. All participants had lesions in the cerebellopontine angle, and none had undergone intracranial surgery or radiotherapy. Patients received surgical treatment, and neurilemmoma was confirmed. Clinical and imaging examination data were collected separately by 2 neurosurgeons. The House-Brackmann facial nerve grading system ${ }^{9}$ was used to evaluate FN function within 7 days after the surgery. This study was examined and approved by the hospital's ethics committee.

\section{Imaging Examination Data}

We performed MRI using a Discovery 750 3.0-T scanner (General Electric). The selection and adjustment of specific scanning parameters for all the sequences were completed under the supervision of the senior doctors in the radiology department of our hospital. The scanning parameters of the DTI sequences included adopting the 8-channel head and neck coil to scan the axial plane and diffuse 30 sensitive gradient directions; TR $4000 \mathrm{msec}$; TE 82.2 msec; scan time 9 minutes; number of slices 40; slice thickness $1.2 \mathrm{~mm}$; gap 0; field of view $200 \mathrm{~mm} \times 200$ $\mathrm{mm}$; acquisition matrix $128 \times 128$; b-value $1000 \mathrm{sec} / \mathrm{mm}^{2}$; and number of excitations 2. Preoperative routine T1weighted imaging, T2-weighted imaging, and sequence scanning were used to obtain 3D anatomical images of the tumor. 3D FIESTA with cycled phase sequences (3D FIESTA-C) was used to accurately select and track the regions of interest (ROIs) of FN visualization using the fol- lowing scanning parameters: TR $4.7 \mathrm{msec}$; TE $2.2 \mathrm{msec}$; slice thickness $0.8 \mathrm{~mm}$; and gap 0 . The imaging data were recorded in DICOM format, thereby facilitating computer processing later.

\section{Visualization of the FN}

MRI data in the DICOM format were uploaded in the 3D Slicer software (http://www.slicer.org/; Harvard University). The late-data format conversion, anatomical images of the tumor, fiber tracking, and 3D visualization of the FN were determined by the software. Each FN was visualized by 2 separate operators at the same time, both of whom were blind to the patients' clinical findings.

The diffusion tensor of the original diffusion-weighted imaging (DWI) data were estimated using the weighted least squares algorithm through the "DWI to DTI estimation" module of the software, and the acquired base image was used as the fixed image. Registration between the 3D FIESTA-C images and the fixed images was performed in the "general registration (brains)" module. The image registration adopts the rigid calibration mode, with 6 degrees of freedom. Scalar mapping operations were applied to the "diffusion tensor scalar measurement" module, and a fractional anisotropy (FA) figure was generated by selective calculation. The "tractography label map seeding" module was adopted for FN tracking and visualization.

Multiple ROI drawings were carried out using the "editor" module before fiber tracking. The ROI was accurately selected according to 3D FIESTA-C imaging. We chose the internal auditory canal (IAC) as the initial seed region, and the FN root exit zone (REZ) as the terminal tracking region (Fig. 1A). The ROI of IAC is the expanding trumpet-shaped zone, while the FN REZ is the area of the pontomedullary sulcus. To increase the success rate of tracking, we usually use the software to add 4 pixels of "shell" to a hand-painted area.

The tracking parameters were as follows: initial tracking space, $0.2 \mathrm{~mm}$; linear measurement threshold value, 0.2 ; FA threshold value, 0.1 ; curvature threshold value, 0.8 ; and shortest fiber bundle length, $5 \mathrm{~mm}$. The tracking parameters were used to track the nerve fiber bundles across the 2 ROIs simultaneously.

Because the ROI is added with a certain thickness of the "shell," the tracking of the fiber bundles unavoidably appears as mixed redundant fiber bundles. Therefore, fiber bundles were appropriately trimmed based on the on the anatomical knowledge of the operator. We use a 3D ROI, which is located within the connection between the IAC and the FN REZ (Fig. 1B). It is tangential to the tumor surface and aims to trim away the fiber bundles outside the region. The portion of the nerve fibers traversing up and down the brainstem, such as the pyramidal tractwhich extends upward and downward definitely-would be pruned. Some inappropriate fiber bundles might appear inside the tumors because of partial volume effects on DTI and the limitations of the FT algorithm. These fiber bundles would also be pruned, being perpendicular to the surface of the tumor and significantly extending into the interior of the tumor.

The development of 3D anatomical imaging of tumors 

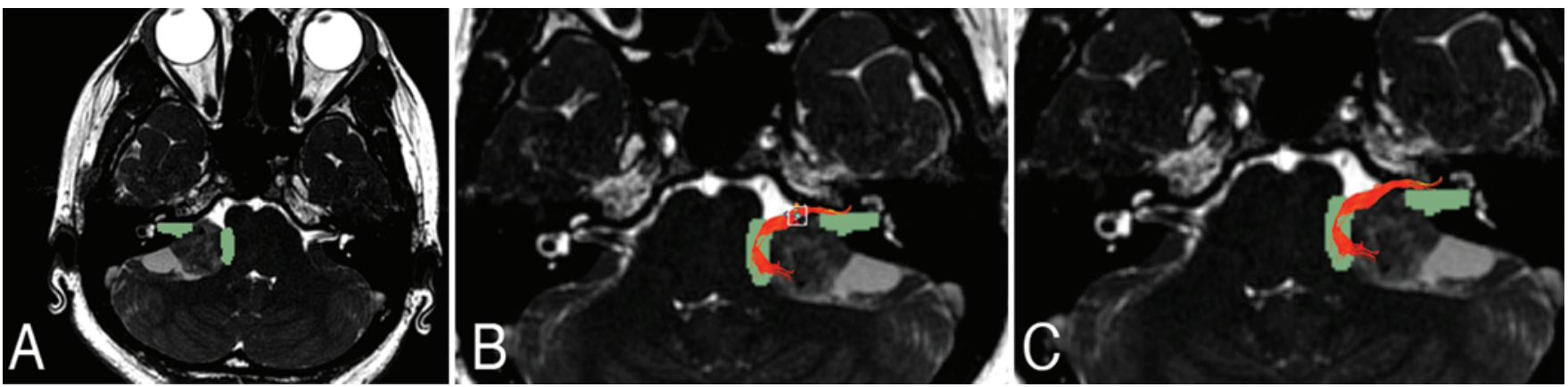

FIG. 1. FN visualization in a patient with the acoustic neuroma on the right side. A: The green translucent parts show 2 ROIs masks (IAC and FN REZ). B: The white square shows the middle ROI. C: FN after proper pruning. Note: panels B and C are both adjusted to show the observation direction from the head to feet (the default observation direction for MRI is from the feet to the head). The upward direction means the upward side of the patient's anatomical position and the subsequent images are shown in this direction, which is not specially noted. Figure is available in color online only.

was based on the enhanced sequences of T1-weighted images and the "editor" module of the software. The anatomical and tracking results of the images of the fiber bundles were fused to determine the course of the FN in a perceptually intuitive way and the relative position between the $\mathrm{FN}$ and tumor.

\section{Assessment of DTI-FT}

Microsurgery was performed by a neurosurgeon (who was blinded to the DTI-FT results) in order to remove the tumor from all 15 patients. The suboccipital retrosigmoid sinus approach ${ }^{12}$ and continuous intraoperative electrophysiological monitoring (the current intensity of the neural probes gradually increased from $0.1 \mathrm{~mA}$ to $1 \mathrm{~mA}$ ) were used during the operation. These approaches stimulated the tumor surface and surroundings in order to probe the existence of any FN in the surgical region, and thus progressively confirmed the FN course. The positional relationship between the FN in the cistern section and the tumor was divided into 6 types: FN in the tumor's anterior upper, anterior middle, anterior lower, superior polar, inferior polar, or posterior portion (Fig. 2). Microscopic images of all patients were recorded using a real-time recording system. Sustained intraoperative FN electrophysiological monitoring was used to determine and record the position and course of the FN and compare the intraoperative results with the preoperative DTI-FT visualization results.

\section{Results}

The median patient age was $43.5 \pm 10.3$ years. Among the participants ( 6 male and 9 female patients), 7 patients had a tumor on the left side of the cerebellopontine angle area, whereas in 8 patients the tumor was on the right side. The major clinical symptoms in these patients before the operation included hearing loss (14 patients), early tinnitus (6 patients), facial numbness (2 patients), blurred vision (2 patients), and walking instability (2 patients). The disease course ranged from 2 months to 20 years (median 2 years).

3D Slicer software was used to develop the 3D anatomical images of the tumor tissues. These images were fused with the 3D FIESTA-C sequence images in order to adjust the tumor model. The images were presented in a translucent manner to better determine the relative positional relationship between the tumor and the surrounding nerve, pons, and cerebellum.

Tumor size was accurately measured (average diameter $31.9 \pm 8.5 \mathrm{~mm}$, range $9-45 \mathrm{~mm}$ ) using software in all patients. The tumor diameters were diameter $\leq 20 \mathrm{~mm}$ (1 patient), $20 \mathrm{~mm}<$ diameter $\leq 30 \mathrm{~mm}$ (6 patients), and diameter $>30 \mathrm{~mm}$ (8 patients) (Table 1$)$.

Among the participants, 11 patients underwent total resection under the microscope, whereas 4 patients underwent subtotal resection. The tumor closely adhered to the FN (4 patients) with a blurred boundary. To protect the function of the FN, a thin layer of tumor tissue was left on the nerve. All patients' FNs achieved anatomical retention. FN electric stimulation, after tumor resection, showed a good nerve response. The patients' postoperative FN function was evaluated according to their House-Brackmann

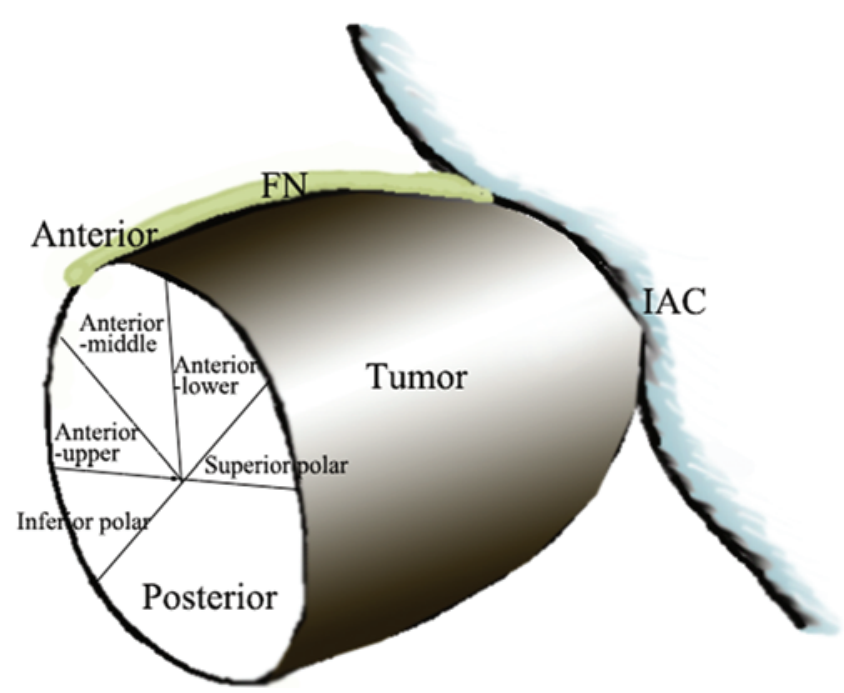

FIG. 2. Relative position between the FN and schwannoma. The line connecting the FN in the IAC and brainstem serves as the axis, and the vertical plane is perpendicular to this line. In the vertical plane, according to the human anatomical location, the quasi-circular cross-section of the tumor is divided into 6 quadrants: the anterior upper, anterior middle, anterior lower, superior polar, inferior polar, and posterior portions of the tumor. Figure is available in color online only. 
TABLE 1. Evaluation of FN function

\begin{tabular}{|c|c|c|c|c|c|c|c|c|c|}
\hline \multirow{2}{*}{$\begin{array}{l}\text { Patient } \\
\text { No. }\end{array}$} & \multirow{2}{*}{$\begin{array}{l}\text { Age } \\
\text { (yrs) }\end{array}$} & \multirow[b]{2}{*}{ Sex } & \multirow{2}{*}{$\begin{array}{l}\text { Tumor } \\
\text { Side }\end{array}$} & \multirow{2}{*}{$\begin{array}{l}\text { Tumor Size } \\
\text { (mm) }\end{array}$} & \multicolumn{2}{|c|}{ F/VN Complex Localization } & \multirow{2}{*}{$\begin{array}{l}\text { Extent of } \\
\text { Resection }\end{array}$} & \multicolumn{2}{|c|}{ F/VN Complex Function (H-B Grade) } \\
\hline & & & & & DTI Fiber Tracking & Interoperation Observation & & Preop & Postop \\
\hline 1 & 54 & M & Left & $27 \times 23 \times 21$ & Anterior upper & Anterior upper & Total & I & II \\
\hline 2 & 26 & $\mathrm{~F}$ & Right & $36 \times 32 \times 37$ & Anterior upper & Anterior upper & Total & I & II \\
\hline 3 & 35 & M & Left & $31 \times 36 \times 43$ & Anterior middle & Anterior middle & Subtotal & II & III \\
\hline 4 & 50 & M & Right & $25 \times 24 \times 25$ & Anterior upper & Anterior upper & Total & I & II \\
\hline 5 & 57 & $\mathrm{~F}$ & Right & $20 \times 19 \times 23$ & Inferior polar & Inferior polar & Total & I & 1 \\
\hline 6 & 31 & $\mathrm{~F}$ & Right & $24 \times 22 \times 20$ & Anterior lower & Anterior lower & Total & I & II \\
\hline 7 & 61 & M & Right & $35 \times 28 \times 37$ & Anterior upper & Anterior upper & Subtotal & I & III \\
\hline 8 & 43 & $\mathrm{~F}$ & Left & $15 \times 10 \times 9$ & Inferior polar & Inferior polar & Total & 1 & 1 \\
\hline 9 & 40 & $\mathrm{~F}$ & Right & $29 \times 34 \times 35$ & Anterior lower & Anterior lower & Subtotal & I & II \\
\hline 10 & 40 & M & Right & $34 \times 42 \times 32$ & Anterior upper & Anterior upper & Total & I & I \\
\hline 11 & 40 & $\mathrm{~F}$ & Left & $25 \times 29 \times 25$ & Anterior upper & Anterior-upper & Total & I & III \\
\hline 12 & 58 & $\mathrm{~F}$ & Right & $17 \times 19 \times 27$ & Anterior lower & Anterior lower & Total & I & 1 \\
\hline 13 & 42 & $\mathrm{~F}$ & Left & $30 \times 34 \times 35$ & Anterior upper & Anterior upper & Total & I & II \\
\hline 14 & 37 & $F$ & Left & $28 \times 25 \times 35$ & Anterior lower & Anterior upper & Total & I & I \\
\hline 15 & 54 & $\mathrm{M}$ & Left & $45 \times 34 \times 35$ & $\mathrm{NA}$ & Anterior upper & Subtotal & III & III \\
\hline
\end{tabular}

F/VN = facial-vestibular nerve; NA = not available.

* Scores according to the House-Brackmann scale. ${ }^{9}$

grades at 7 days after the operation, and grade comparison was also carried out for pre- and postoperative FN function. The results show good retention of function (Table 1).

The preoperative MRI data for the 15 patients with acoustic neuroma were processed using 3D Slicer software (based on DTI-FT technology). Additionally, operations for fiber tracking and 3D visualization of the FN were carried out by 2 neurosurgeons separately. The visualization results were compared to verify the repeatability of the operation. The neural fasciculus courses that simultaneously passed through 2 ROIs (the IAC and REZ regions of FN) were successfully tracked and reconstructed in 14 patients. The fasciculus of 1 patient was short and passed only $1 \mathrm{ROI}$ of the REZ region of FN, and thereby failed to be tracked and reconstructed.

In patients treated with DTI-FT technology for FN visualization $(\mathrm{n}=14)$, comparison validations were carried out for the visualization results, intraoperative actual course, and its positional relationship with the tumor by microscopic observation and direct FN electrophysiological stimulation. The results from the comparison validation demonstrated that the FN visualization results were consistent with the actual situation of the operation in 13 of 14 patients (92.9\%) (Figs. 3 and 4), whereas in 1 patient the results were inconsistent. Among the consistent cases, the FN was located on the anterior upper side $(n=7)$, anterior middle portion $(n=1)$, anterior lower portion $(n=3)$, or inferior polar side $(n=2)$ (Table 1).

\section{Discussion}

Since the late 1990s, DTI technology has been used to study cerebral white matter fibers. ${ }^{13}$ However, in the last decade, there has been tremendous progress in DTI-FT technology for studying the important fiber bundles in the cerebral white matter such as the pyramidal tract, arcuate fasciculus, and optic radiation. ${ }^{3}$ In 2007, Kabasawa et al. ${ }^{10}$ conducted DTI-FT visualization research on the cranial nerves, including the optic nerve, trigeminal nerve, and auditory nerve in healthy individuals. ${ }^{8}$ In patients with acoustic neuroma, imaging is difficult due to the compression of the nerves by the tumor. Different studies have reported the use of DTI-FT in patients with acoustic neuroma; ${ }^{1,5,14,20}$ however, we have repeated these experiments on the basis of the above reported literature, but the obtained reconstruction results are not ideal.

\section{DTI-FT of the FN}

In patients with acoustic neuroma, tumor compression and push result in 3 different configurations of the nerves: parallel; wrapped in the arachnoid membrane; or adherence to one another. In our study, we found that the spatial resolution of DTI was not able to characterize the FN in the vestibular and cochlear nerves. FN visualization, using this technology, mainly included the facial-vestibular nerve complex of the FN, vestibular nerve, and even the cochlear nerve. ${ }^{1}$ It is also very difficult to separate the FN and vestibular nerve in most microsurgery operations for acoustic neuroma. Therefore, the FNs in our study actually demonstrated the course of the facial-vestibular nerve complex whether in tractography or surgery.

In this study, a nerve fiber course that completely crossed 2 ROIs was observed in 14 patients (93.3\%), thereby demonstrating good repeatability of the technology. The course position and direction of the suspected fiber bundles of the FN was also in line with the common positions and FN courses. However, in 1 patient, the course of the FN were not successfully tracked. The tumor was found to be a solid cyst, and the cyst's fluid and substantial part were mixed, thereby affecting the spatial resolution of the DTI images. In addition, the tumor had a big vol- 

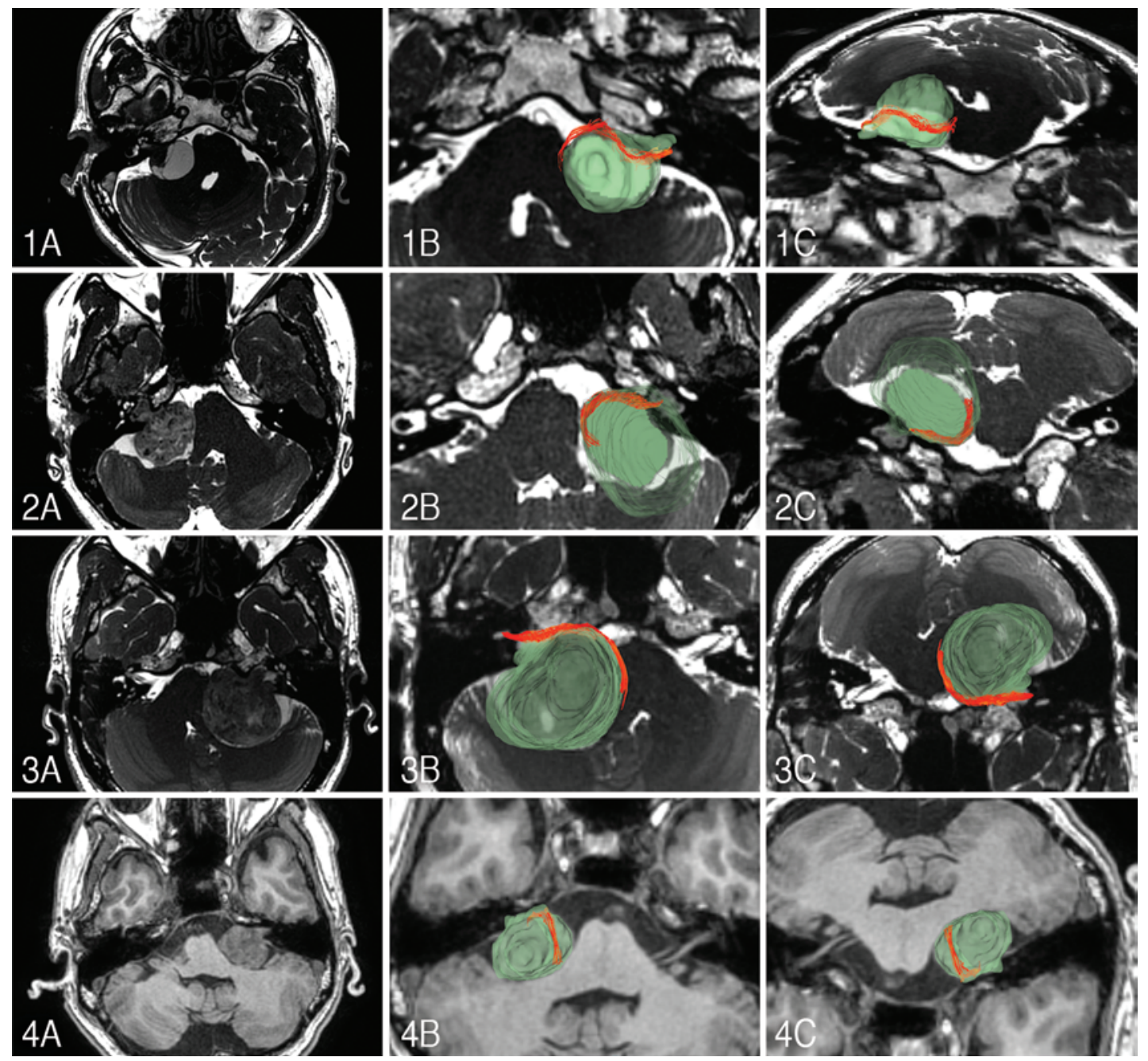

FIG. 3. Four cases of FN visualization and the intraoperative observations of a patient with acoustic neuroma. The green translucent parts shows a 3D anatomical model of the tumor, and red parts shows the reconstructed FN. The FN is located in the anterior upper portion of the tumor (1); the anterior lower portion of the tumor (2); the anterior middle portion of the tumor (3); and the anterior upper part of the tumor (4). Figure is available in color online only.

ume, where the FN was compressed to form a mushroom umbrella-like structure that adhered closely to the tumor wall, and there was no obvious boundary microscopically. Electrical stimulation showed that the nerve was present in the anterosuperior side of the tumor and partially fused with the tumor wall. Thus, only a short, discontinuous fiber bundle that went through a single ROI could be tracked. In schwannoma patients $(\mathrm{n}=8)$, Taoka et al. ${ }^{18}$ reported that 1 patient whose FN visualization result was inconsistent with the actual position had a tumor that was mostly cystic. From these studies, we inferred that the cystic tumor may affect the tracking of the fasciculus. However, Gerganov et al. reported that the DTI-FT results of the FN in 2 of 22 patients with large vestibular schwannomas were not in accordance with the actual course, and these 2 patients had no important differences with the others in terms of their tumor characteristics. ${ }^{5}$ Thus, further studies are required to establish the relationship between DTI-FT and the tumor characteristics.

In patients with acoustic neuroma, the course of the
FN's cistern section was not constant due to tumor compression. According to the anatomical data obtained from a large number of samples, the majority of FNs were located in the ventral portion and few in the superior, inferior polar, and posterior portions of the tumor. ${ }^{17}$ In this study, we found that the FN visualization results were identical to the actual position in the operation and the location distribution (according to the anatomical statistical data) in 13 patients (92.9\%). However, in 1 patient, visualization of the FN was not consistent with the actual location. The possible reasons for this were the FA value of the tumor's substantial part, the protein structure of the cyst fluid, and low FA threshold value. Consequently, some of the FA values of the non-fiber bundle structure were incorrectly tracked, which resulted in the deviation in the visualization course of the fiber bundles. In further study, reduction in interference against fasciculus tracking can be obtained by selecting more accurate ROIs and adjusting the FA threshold value.

As shown in Fig. 4, we can see that the FN was in good 

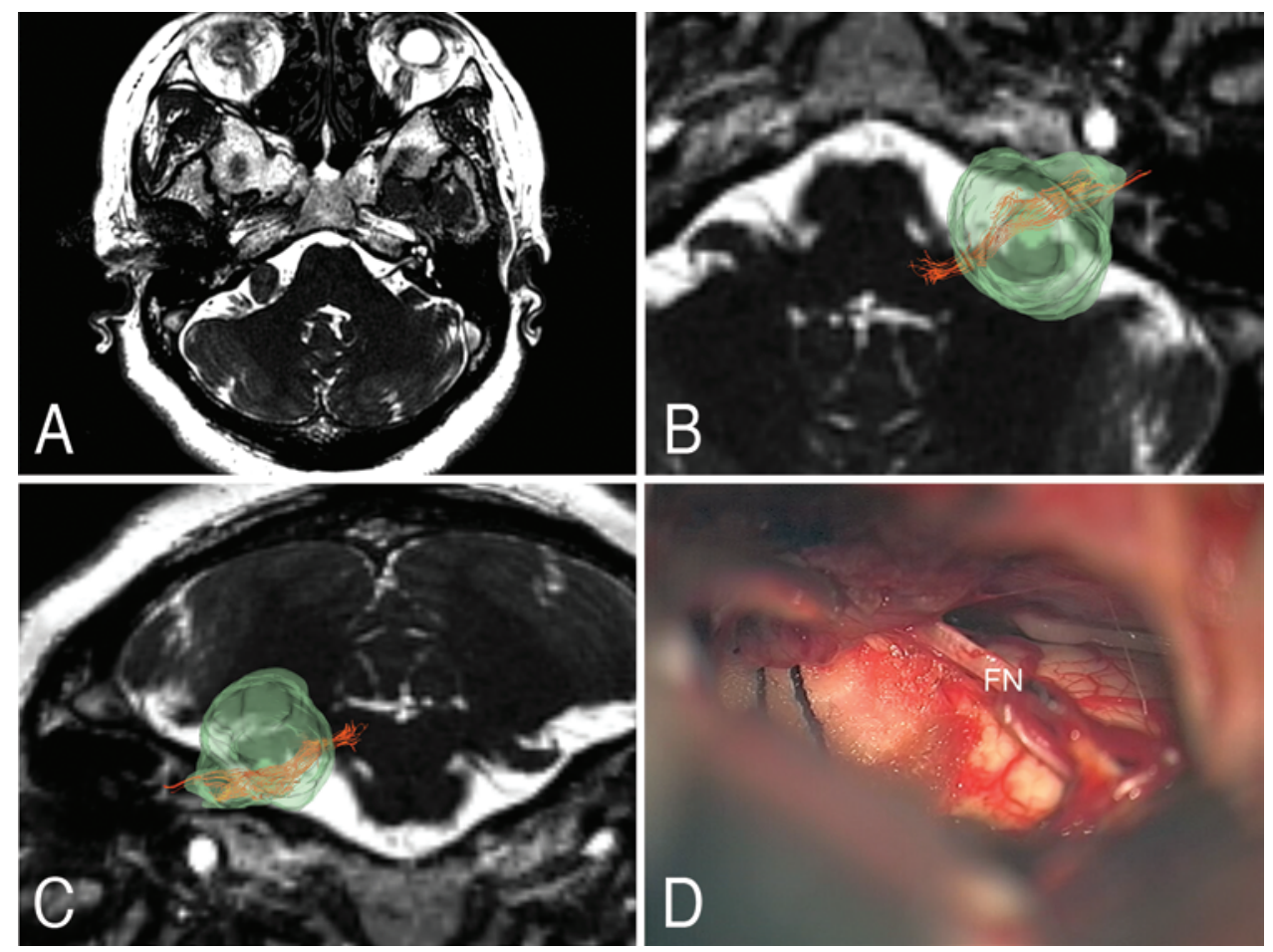

FIG. 4. Reconstruction of the FN and intraoperative observations of a patient with acoustic neuroma on the right side. A: Axial 3D FIESTA-C sequence image showing that the tumor (small volume) is solid. B and C: The red part shows that the FN is located in the inferior polar of tumor. D: Intraoperative observations showing the course of the FN below the tumor, which is consistent with the DTI-FT reconstruction results. Figure is available in color online only.

condition, as only a part of it was compressed by the tumor; however, the density of the fiber bundle after visualization was relatively low. From this study, it seems that there was no correlation between the shape of the fiber bundles (after visualization) and the actual shape of the FN as observed in the operation. In the fiber-tracking algorithm, fiber tracking is compromised due to the destruction of the FN by the infiltrating tumor. However, due to the interference of CSF, the FN exposed to tumor capsule will influence the resolution of the DTI signals, thereby significantly reducing the number of fiber bundles tracked and reconstructed. Further investigations will be required to determine if such phenomena will have statistical significance for clinical applications.

Therefore, the high feasibility of the reconstructed FN using DTI-FT technology for patients with acoustic neuroma can be effectively used for clinical applications. The combination of DTI-FT with intraoperative electrophysiological monitoring enhances the protection of the FN.

\section{Parameter Selection for DTI-FT}

The accuracy of the FN visualization mainly depends on high-quality DTI data acquisition and the selection of appropriate fiber-tracking parameters.

Due to the presence of the FN in CSF (with a reduced diameter) and its deformation due to tumor compression, a high spatial resolution and signal-to-noise ratio in imaging is required to clearly determine the position and course of the FN. We adopted 1.5-T MR to acquire DTI data; however, visualization of any fasciculus was not carried out as it would have resulted in the wrong tracking of the fiber course. Later, we used 3.0-T MRI with high field intensity for scanning and obtained satisfying tracking results. MRI with high field intensity provides higher precision and resolution ratio of the images. Thus, applying high field intensity MRI results in detailed tracking of the fasciculus. Chen et al. also affirmed that higher field strengths were highly advantageous, but its importance cannot be overemphasized. ${ }^{1}$ The study demonstrated that adjusting the layer thickness to thin improves the spatial resolution; however, it tends to reduce the signal-to-noise ratio at the same time. In similar foreign research, the choice of thickness is $1.2 \mathrm{~mm}$ or $3.0 \mathrm{~mm} .{ }^{1,10,14,18,19} \mathrm{In}$ earlier experiments, we have used these 2 kinds of thicknesses for DTI data acquisition in order to compare the later tracking results. The comparison found that the 1.2-mm group has higher FN fiber continuity, and the number of cases in line with the satisfactory results of the clinical application is more than that of the 3.0-mm group. Heros and Bhatia also think that thinner slices cause less image distortion and signal loss, which may have accounted for the better visualization of the facial nerves. ${ }^{7}$ Based on this understanding, we chose a thickness of $1.2 \mathrm{~mm}$ for the DTI data acquisition in later experiments. But, the drawback of the obtained result is that there are fewer earlier experiment samples, but this has not been statistically verified.

The 3D Slicer application software has gained significant attention in imaging data and image postprocessing. ${ }^{4,11}$ For selecting the fiber-tracking parameters, the FA threshold value influences the results of FN tracking and 
visualization. A study reported that an FA threshold value of 0.1 FA produces satisfactory visualization results, ,,14,18 and a 0.2-FA threshold results in the successful visualization of the FN. ${ }^{1}$ In this study, we selected an FA threshold value of 0.1 for tracking. This value was significant for providing successful tracking and reconstructing the compressed acoustic nerves.

In this study, it was evident that erroneous fibers would be tracked only when the IAC or FN REZ was used as the ROI, and tracking the wrong fibers was reduced when both IAC and FN REZ were used to for tracking the ROI zone. For ROI positioning, we selected the 3D FIESTA-C sequence as the positioning benchmark to dissect the image. 3D FIESTA-C is a rapid, steady-state imaging sequence in which 3D scanning using 1-mm-thick layers can be conducted. The 3D FIESTA-C sequence has the following advantages: high spatial resolution and high contrast ratio between the CSF and cranial nerves; short scanning time; stable image quality; and it provides more elaborate anatomical detail on the display of the skull base structure. ${ }^{2}$ In healthy individuals, 3D FIESTA-C is used to observe the whole course of the cistern section. In this study, the FN was not clearly recognized in 14 patients; however, part of the fasciculus course in the adjacent REZ of the FN was observed in only 1 patient (15-mm diameter tumor). Thus, we think that in patients with acoustic neuroma, especially patients harboring larger-diameter schwannomas, image identification of the FN course using 3D FIESTA-C is difficult. However, due to its high resolution and thinslice scanning characteristics, it is helpful for IAC and FN REZ zone identification on DTI. Thus, we used the 3D FIESTA-C sequence as an auxiliary for precisely setting the ROIs for FN fiber tracking. ${ }^{1}$

\section{Study Limitations}

In this study, visualization of the FN showed only the position and course; however, its concrete, free, or adhesive form and the degree of adhesion were not specified.

This retrospective, single-center study evaluated the role of DTI-FT for clinical applications. However, to establish the reliability and accuracy of FN tracking visualization, multicenter clinical research (with a large sample capacity) is required. Additionally, the specific parameters of the scanning sequence need further adjustment and optimization, and further improvements are required in the tracking algorithm for the fiber bundles.

The results of this study and other related studies have demonstrated the feasibility of DTI-FT for FN visualization. However, these results need to be studied in light of the actual surgery. Additionally, the technology requires further research in order to develop the technology as a standard scheme for FN visualization.

\section{Conclusions}

The success rate of visualizing the FN by DTI-FT was 93.3\% (14 of 15 patients). The accuracy rate of the visualization results of the FN was $92.9 \%$ (13 of 14 patients). DTI-FT, in combination with FN electrophysiology monitoring during operation, provides better protection of $\mathrm{FN}$ functions.

\section{Acknowledgments}

This study is supported by National Natural Science Foundation of China (no. 81271365).

\section{References}

1. Chen DQ, Quan J, Guha A, Tymianski M, Mikulis D, Hodaie M: Three-dimensional in vivo modeling of vestibular schwannomas and surrounding cranial nerves with diffusion imaging tractography. Neurosurgery 68:1077-1083, 2011

2. Cheng YS, Zhou ZR, Peng WJ, Tang F: Three-dimensionalfast imaging employing steady-state acquisition and T2weighted fast spin-echo magnetic resonance sequences on visualization of cranial nerves III - XII. Chin Med J (Engl) 121:276-279, 2008

3. Ciccarelli O, Toosy AT, Parker GJ, Wheeler-Kingshott CA, Barker GJ, Miller DH, et al: Diffusion tractography based group mapping of major white-matter pathways in the human brain. Neuroimage 19:1545-1555, 2003

4. Fedorov A, Beichel R, Kalpathy-Cramer J, Finet J, FillionRobin JC, Pujol S, et al: 3D Slicer as an image computing platform for the Quantitative Imaging Network. Magn Reson Imaging 30:1323-1341, 2012

5. Gerganov VM, Giordano M, Samii M, Samii A: Diffusion tensor imaging-based fiber tracking for prediction of the position of the facial nerve in relation to large vestibular schwannomas. J Neurosurg 115:1087-1093, 2011

6. Gupta S, Mends F, Hagiwara M, Fatterpekar G, Roehm PC: Imaging the facial nerve: a contemporary review. Radiol Res Pract 2013:248039, 2013

7. Heros RC, Bhatia R: Editorial: vestibular schwannoma and the facial nerve. J Neurosurg 116:695-696, 2012

8. Hodaie M, Quan J, Chen DQ: In vivo visualization of cranial nerve pathways in humans using diffusion-based tractography. Neurosurgery 66:788-796, 2010

9. House JW, Brackmann DE: Facial nerve grading system. Otolaryngol Head Neck Surg 93:146-147, 1985

10. Kabasawa H, Masutani Y, Aoki S, Abe O, Masumoto T, Hayashi N, et al: 3T PROPELLER diffusion tensor fiber tractography: a feasibility study for cranial nerve fiber tracking. Radiat Med 25:462-466, 2007

11. Kikinis R, Pieper S: 3D Slicer as a tool for interactive brain tumor segmentation. Conf Proc IEEE Eng Med Biol Soc 2011:6982-6984, 2011

12. Mazzoni A, Calabrese V, Danesi G: A modified retrosigmoid approach for direct exposure of the fundus of the internal auditory canal for hearing preservation in acoustic neuroma surgery. Am J Otol 21:98-109, 2000

13. Pierpaoli C, Jezzard P, Basser PJ, Barnett A, Di Chiro G: Diffusion tensor MR imaging of the human brain. Radiology 201:637-648, 1996

14. Roundy N, Delashaw JB, Cetas JS: Preoperative identification of the facial nerve in patients with large cerebellopontine angle tumors using high-density diffusion tensor imaging. J Neurosurg 116:697-702, 2012

15. Samii M, Gerganov VM, Samii A: Functional outcome after complete surgical removal of giant vestibular schwannomas. J Neurosurg 112:860-867, 2010

16. Samii M, Matthies C: Management of 1000 vestibular schwannomas (acoustic neuromas): the facial nerve-preservation and restitution of function. Neurosurgery 40:684695, 1997

17. Sampath P, Rini D, Long DM: Microanatomical variations in the cerebellopontine angle associated with vestibular schwannomas (acoustic neuromas): a retrospective study of 1006 consecutive cases. J Neurosurg 92:70-78, 2000

18. Taoka T, Hirabayashi H, Nakagawa H, Sakamoto M, Myochin K, Hirohashi S, et al: Displacement of the facial nerve course by vestibular schwannoma: preoperative visualization 
using diffusion tensor tractography. J Magn Reson Imaging 24:1005-1010, 2006

19. Wai Y, Chu J, Wang C, Lin Y, Lin G, Wan Y, et al: An integrated diffusion map for the analysis of diffusion properties: a feasibility study in patients with acoustic neuroma. Acad Radiol 16:428-434, 2009

20. Zhang Y, Chen Y, Zou Y, Zhang W, Zhang R, Liu X, et al: Facial nerve preservation with preoperative identification and intraoperative monitoring in large vestibular schwannoma surgery. Acta Neurochir (Wien) 155:1857-1862, 2013

\section{Disclosures}

The authors report no conflict of interest concerning the materi- als or methods used in this study or the findings specified in this paper.

\section{Author Contributions}

Conception and design: Zhang, Song. Acquisition of data: Song, Hou. Analysis and interpretation of data: Hou, Sun. Drafting the article: Chen. Critically revising the article: Chen. Reviewed submitted version of manuscript: Xu. Statistical analysis: Chen. Administrative/technical/material support: Xu. Study supervision: Huang.

\section{Correspondence}

Jun Zhang, Department of Neurosurgery, PLA General Hospital, Beijing 100853, China. email: junzhang301@163.com. 